\title{
Performance Analysis of FBMC-OQAM based 5G Wireless System using PAPR
}

\author{
Parnika Kansal \\ Department of Electronics Engineering, \\ School of Engineering, \\ Harcourt Butler Technical University \\ Kanpur, Uttar Pradesh, India
}

\author{
A. K. Shankhwar \\ Associate Professor \\ Department of Electronics Engineering \\ School of Engineering, HBTU Kanpur \\ Uttar Pradesh, India
}

\begin{abstract}
This research work has an area of interest related to $5 \mathrm{G}$ technology driven by the concepts and formulations of different $5 \mathrm{G}$ forums developed across countries. This research surveys the idea of $5 \mathrm{G}$ communication technique with its conquest for bandwidth versus user. In this scenario the different forum have worked with different streams of frequencies ranging in Giga hertz. However in this research paper the main study and discussion focuses on power problem highlighted in $5 \mathrm{G}$. This research work cares about FBMC 5G technique which remains a powerful contender of $5 \mathrm{G}$ with a disadvantage of power, the power problem is formulated using PAPR term and its reduction is also applied based on different techniques of PAPR reduction which are applicable to $4 \mathrm{G}$ LTE technique. Thus after careful observations the research paper recommends DSLM as a powerful technique of PAPR reduction $5 \mathrm{G}$ systems with the least PAPR values.
\end{abstract}

\section{General Terms}

Wireless Communication, Next Generation Mobile Network Telecommunication Standards

\section{Keywords}

FBMC, Clipping, SLM, DSLM, PHYDAS, PAPR, Emphatic, OQAM, 5G, ITU

\section{INTRODUCTION}

The advancement of mobile broadband wireless to the fifth generation is driven by expanded limit requests, enhanced throughput and extra utilize cases for wireless access where all things that can be associated will be associated in a seamless manner. The International Telecommunications Union Radio communication Sector (ITU-R), in close joint effort with different partners including the worldwide mobile industry, has set out on characterizing the procedure, course of events and deliverables for the up and coming generation of IMT frameworks, called IMT-2020, to understand this future vision of mobile broadband communications.5G is connected with the following stride of IMT (i.e., IMT-2020), for which initial planning is as of now under way in the ITU. Also, various different changes at end to-end framework will be a piece of $5 \mathrm{G}$ advancement, both in the Radio Access Network (RAN) and centre network. $5 \mathrm{G}$ is the term that is being connected in the market to frameworks past IMT-Advanced (i.e., past LTEAdvanced and WMAN-Advanced).In its different white papers on the subject, 4G Americas has expressed that while past generations have been distinguished by a noteworthy new innovation step, for example, the meaning of another air interface, the desire is that $5 \mathrm{G}$ will be drawn closer from an end-to-end framework point of view and incorporate real innovation steps both in the RAN and centre network. $3 \mathrm{G}$ and $4 \mathrm{G}$ advancements are principally centred on the mobile broadband use case, giving upgraded framework limit and offering higher information rates. This concentration will obviously proceed later in $5 \mathrm{G}$ era, with limit and information rates being driven by administrations, for example, video.

Yet, the future likewise will be a great deal more than only improvements to the "ordinary" mobile broadband use case. Future wireless networks ought to offer wireless access to anybody and anything. Accordingly, later on, wireless access will go past people and extend to serve any entity that may profit by being associated. This vision regularly is alluded to as Internet of things (IoT), Networked society, Machine to Machine Communications or Machine driven communications. North American administrators' best clients are no more extended people; they're progressively machines, for example, smart utility meters, digital signage and vehicle infotainment systems [1].

Enhanced Multicarrier Technology for Professional Adhoc and Cell-based Communications (EMPhAtiC) assignment develops tremendously bendy and effective filter-bank processing structure and channel estimation, equalization and synchronization functionalities wanted therein. Moreover, feasibility of filter bank based multicarrier schemes in some distinct environments (relays, multihop, cooperative), the place synchronization is difficult to keep, will be evaluated. As specialized prerequisites over currently existing technologies $(4 \mathrm{G})$ records the accompanying:

1. 1000 times higher mobile information volume per region,

2. 10 to 100 times higher average client information rate,

3. 10 to 100 times higher number of associated devices,

4. 10 times longer battery life for low power devices,

5. times reduced end-to-end latency [2].

There is no one of a kind definition (yet) for $5 \mathrm{G}$.However, a general agreement is working around the possibility that $5 \mathrm{G}$ is just combination of a few technologies, scenarios and use cases as opposed to the development of another single radio access technology $[3,4]$.

The rest of the paper is organised as follows-

Section II picks up the brief essentials of various ongoing $5 \mathrm{G}$ research projects, Section III reviews the various waveform contenders for $5 \mathrm{G}$, their comparison, and also compares the various mobile wireless generations, Section IV covers the FBMC waveform contender for $5 \mathrm{G}$ and its mathematical model, Section V gives the results of simulations and the study done on 5G, and finally Section VI gives the conclusion. 


\section{KEY RESEARCH PROJECTS OF 5G}

This section overviews usually European Union seventh Framework Programme (FP7) Future Networks Cluster Radio access and Spectrum tasks managing a giant part of European $5 \mathrm{G}$ research hobbies. New research applications and moves are coordinated through Horizon 2020 and 5G Infrastructure Public Private Partnership (5GPPP)[5]. It also reviews the 4G Americas project.

Mobile and wireless communications Enablers for the Twenty-twenty information Society (METIS) is the most important FP7 5G project (29 partners) whose goal is to create basis for $5 \mathrm{G}$ systems and act as a consensus builder towards standardization. METIS has recognized some test instances and eventualities to demonstrate and handle the important thing challenges $5 \mathrm{G}$ will face.

The scenarios are listed as:

1. 'Amazingly fast',

2. 'Great provider in a crowd',

3. 'Nice expertise follows you',

4. 'Super actual-time and reliable connections', and

5. 'Ubiquitous things communicating [6,7].

METIS 5G Requirements are-

1. Data Rate-1-10 Gbps (resp 100s of Mbps)

2. Capacity-36TB/month/user (resp 500GB)

3. Spectrum-Higher frequencies and flexibility

4. Energy- 10\% of today's consumption

5. Latency Reduction- 1ms (eg.tactile internet)

6. D2D capabilities- NSPS, ITS, resilience

7. Coverage-20 dB of LTE (eg. sensors)

8. Battery- $\sim 10$ years

9. Devices per area- 300.00 per access node.

Fifth generation Non-Orthogonal Waveforms for Asynchronous Signaling (5GNOW) challenge proposes a scalable and effective air interface that gives up on strict orthogonality and synchronism principles followed in former new release networks. Universal filtered multi-carrier (UFMC), filter bank multi-carrier (FBMC) and generalized frequency division multiplexing (OFDM) are few examples of promising waveforms that may outperform orthogonal frequency division multiplexing (OFDM) in 5G context. In addition to multicarrier waveform design itself, 5GNOW addresses such features as a unified body structure, filtering, sparse signal processing, robustness, and very low latency transmissions $[8,9]$.

Enhanced Multicarrier technological for authentic Adhoc and cell based Communications (EMPhAtiC) project develops enormously flexible and potent filter-bank multi carrier structures and channel estimation, equalization and synchronization functionalities wanted therein. Moreover, feasibility of filter bank established multicarrier schemes in some unique environments (relays, multihop, cooperative), the position synchronization is rough to hold, will be evaluated. The Emphatic project officially started on September 1st, 2012, and has finished on February 28, 2015 [10].
The 4G Americas in its more than a few white papers on the subject, has stated that while past generations had been recognized with the aid of a fundamental new technology step, such as the definition of a new air interface, the expectation is that $5 \mathrm{G}$ might be approached from an end-to-end approach standpoint and incorporate principal science steps each within the RAN and core network. 5G is related to the next step of IMT (i.e., IMT-2020), for which initial planning is currently underneath approach within the ITU. Additionally, a quantity of other changes subsequently-to-end process can be a part of 5G evolution, both within the Radio access network (RAN) and core network. 4G Americas believes that the mobile industry is capable of extending mobile services into spectrum bands in the range above $6 \mathrm{GHz}$ to gain additional bandwidth. In the coming years, the U.S. government has identified 1,000 $\mathrm{MHz}$ of federal spectrum that could be made available for commercial use on a shared basis. The ITU-R has recognized three main utilization scenarios for $5 \mathrm{G}$ :

1. Enhanced mobile broadband

2. Ultra-reliable and low latency communications

3. Massive machine type communications

The mission of $4 \mathrm{G}$ Americas is to recommend for and foster the advancement and full capabilities of the LTE mobile broadband technological know-how and its evolution past to $5 \mathrm{G}$, for the duration of the ecosystem's networks, services, functions and wirelessly related contraptions within the Americas. 4G Americas, the voice of $5 \mathrm{G}$ for the Americas, is invested in leading 5G development for the Americas and keeping the current world innovation lead in North America with LTE technology.

\section{COMPARISON OF DIFFERENT WIRELESS GENERATIONS \& WAVEFORM CANDIDATES OF 5G}

The basic idea of communication systems involving different wired and wireless devices includes a combination of different spectrum working altogether to bring information pieces in a rapid and convenient manner. Different generations of spectrum decided and allotted by the ITU and different communication forums brings the change that is needed for the support of information interchange in the form of text, video, multimedia, voice and internet. Figure 1 is a description of different generations of communication spectrum allotted so far that comes hand to hand in market at a very convenient price.

\subsection{G VS 2G V 3G VS 4G V 5G}

The following figure describes the different generations of communication spectrum allots so far that comes hand to hand in market at a very convenient price and the time period. 


\begin{tabular}{|c|c|c|c|c|c|}
\hline $\begin{array}{l}\text { Technology } \\
\text { /Features }\end{array}$ & $1 G$ & $2 / 2.5 G$ & $3 G$ & $4 G$ & $5 G$ \\
\hline $\begin{array}{c}\text { Start/ } \\
\text { Deployment }\end{array}$ & $1970 / 1984$ & $1980 / 1999$ & 1990/2002 & $2000 / 2010$ & $2010 / 2015$ \\
\hline $\begin{array}{c}\text { Data } \\
\text { Bandwidth }\end{array}$ & 2 kbps & 14.4-64 kbps & 2 Mbps & $\begin{array}{l}200 \text { Mbps to } \\
1 \text { Gbps for low } \\
\text { mobility }\end{array}$ & $\begin{array}{l}1 \text { Gbps and } \\
\text { higher }\end{array}$ \\
\hline Standards & AMPS & $\begin{array}{c}\text { 2G: TDMA, } \\
\text { CDMA, GSM } \\
2.5 \mathrm{G}: \text { GPRS, } \\
\text { EDGE,IxRTT }\end{array}$ & $\begin{array}{l}\text { WCDMA, } \\
\text { CDMA-2000 }\end{array}$ & $\begin{array}{l}\text { Single } \\
\text { unified } \\
\text { standard }\end{array}$ & $\begin{array}{l}\text { Single } \\
\text { unified } \\
\text { standard }\end{array}$ \\
\hline Technology & $\begin{array}{l}\text { Analog } \\
\text { cellular } \\
\text { technology }\end{array}$ & $\begin{array}{l}\text { Digital cellular } \\
\text { technology }\end{array}$ & $\begin{array}{l}\text { Broad } \\
\text { bandwidth } \\
\text { CDMA, IP } \\
\text { technology }\end{array}$ & $\begin{array}{l}\text { Unified IP and } \\
\text { seamless } \\
\text { combination } \\
\text { of broadband, } \\
\text { LAN/WAN/ }\end{array}$ & $\begin{array}{l}\text { Unified IP and } \\
\text { seamless } \\
\text { combination } \\
\text { of } \\
\text { broadband, }\end{array}$ \\
\hline
\end{tabular}

Fig1- Comparision of different wireless generations [16]

\subsection{Waveform Contenders for 5G}

OFDM is a well-known, completely studied and closely applied waveform design precept. Simply to call two, both 4G (LTE and its evolutions so far) and IEEE 802.11 (Wi-Fi) use OFDM as basic signal format for carrying the data by way of the air. Even as having many best features, OFDM has a foremost characteristic making it less appealing for the mobile conversation process to come back (more often than not mentioned5G). Each and every single subcarrier in an OFDM system is shaped making use of an oblong window in time area leading to sinc shaped subcarriers in frequency domain (making use of windows with tender transitions at both improves on this, nonetheless, introducing intercarrier interference). So, one of the celebrated characteristic of OFDM (dividing the spectrum into more than one parallel orthogonal sub-bands with easiest viable effectivity) applies only in case of best situations. These circumstances are for example best frequency synchronization and tight time alignment within the duration of the cyclic prefix. LTE is able to attain this by way of applying a closed loop ranging mechanism and demanding very strict oscillator standards. The one devices will not be allowed to transmit information so long as the base station will not be satisfied with appreciate to the time and frequency alignment of their transmissions. Concluding, it is apparent that the air interface of $5 \mathrm{G}$ very doubtless wants to be higher perfect to a diminish measure of synchronization as demanded with the aid of OFDM, as OFDM suffers from robust intercarrier interference in this case. Consequently, in contemporary years a substitute to OFDM has been heavily promoted: Filter bank multicarrier (FBMC) [11,12].

\section{1.) FBMC(Filter Bank Multi carrier)}

FBMC has gained a high measure of interest as a potential 5G waveform candidate. This waveform scheme presents many benefits. In lots of approaches FBMC has many similarities to CP-OFDM, OFDM using a cyclic prefix which is used because the $4 \mathrm{G}$ waveform. Rather of filtering the entire band as in the case of OFDM, FBMC filters each sub-provider for my part. FBMC does now not have a cyclic prefix and thus it is ready to provide an awfully high degree of spectral efficiency. The subcarrier filters are very slender and require lengthy filter time constants. Quite often the time steady is four instances that of the elemental multicarrier image length and for this reason, single symbols overlap in time. To reap orthogonality, offset-QAM is used because the modulation scheme, so FBMC just isn't orthogonal with recognize to the elaborate plane [13].

\section{2.) Universal Filter Bank Multi Carrier}

This $5 \mathrm{G}$ waveform can also be regarded as an enhancement of CP-OFDM. It differs from FBMC in that as a substitute of filtering every subcarrier personally, UFMC splits the sign into a number of sub-bands which it then filters. UFMC does not need to use a cyclic prefix, even though one can be utilized to fortify the inter-symbol interference protection.

\section{3.) GFDM}

Generalised Frequency Division Multiplexing is a flexible multi-carrier transmission procedure which bears many similarities to OFDM. The essential difference is that the carriers are not orthogonal to one another. GFDM provides better manipulate of the out-of-band emissions and reduces the peak to average power ratio, PAPR. Each of these problems are the fundamental drawbacks of OFDM technology.

\section{4.) BFDM-}

In bi-orthogonal frequency division multiplexing (BFDM) symbols can be perfectly recovered by using bi-orthogonality approach. Bi-orthogonality is a weaker form of orthogonality, here transmit and receive pulses are no longer orthogonal to each other.

\section{MATHEMATICAL MODELLING OF FBMC-OQAM SYSTEM}

In a FBMC-OQAM process we use complex symbols that are modulated situated on OQAM and then transmitted [14] .The $\mathrm{M}$ elaborate input symbols with $\mathrm{N}$ sub-carriers at the transmitter may also be written as-

$C_{m, n}=R_{m}^{n}+j I_{m}^{n}, 0 \leq n \leq N-1,0 \leq m \leq M-$ 1

where, $R_{m}^{n}$ and $I_{m}^{n}$ re the real and imaginary parts of the $m^{t h}$ symbol on the $n^{\text {th }}$ sub-carrier, respectively. The input symbols in $m^{\text {th }}$ data block can be grouped as a vector as shown below-

$$
\begin{gathered}
\boldsymbol{C}_{\boldsymbol{m}}=\left(\boldsymbol{c}_{\boldsymbol{m}, \mathrm{o}}+\boldsymbol{C}_{\boldsymbol{m}, \mathbf{1}}+\cdots \ldots \ldots \ldots \boldsymbol{C}_{\boldsymbol{m}, \boldsymbol{N}-\mathbf{1}}\right)^{\boldsymbol{T}} \mathbf{0} \leq \mathrm{n} \\
\leq \mathrm{N}-1(2)
\end{gathered}
$$

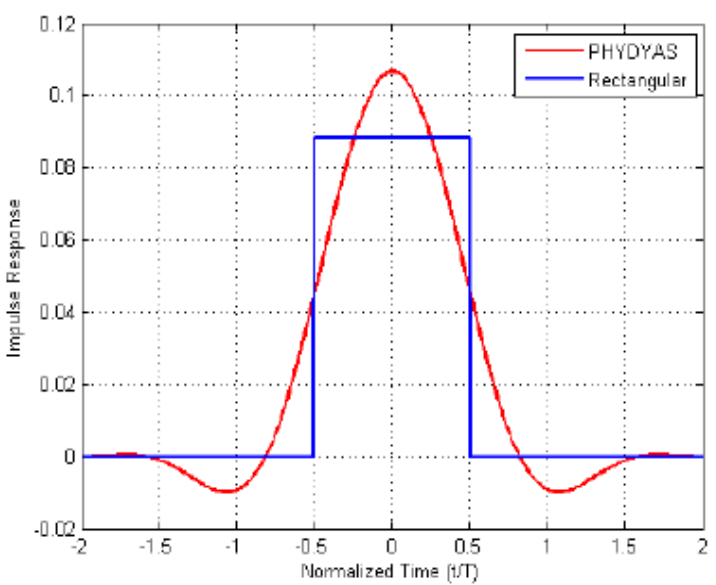

Fig 2- Impulse response of PHYDAS AND Rectangular filters [17]

In OQAM, the real and imaginary parts of the symbols are time staggered with the aid of $T / 2$, where $T$ is the symbol period. It is finished in this sort of approach that, for every two adjacent sub-carriers, the timing offset of $T / 2$ is presented on the real part of the former symbol whereas it will be on the 
imaginary part of the latter one. Then, these symbols are handed and passed through a bank of synthesis filters to get modulated with $\mathrm{N}$ sub-carriers the place the spacing between each two sub-carriers is $1 / \mathrm{T}$ [15].

$(\mathrm{t})=\sum_{n=0}^{N-1} \sum_{m^{\prime}=0}^{2 M-1} a_{m^{\prime}, n} h\left(t-m^{\prime} T / 2\right) e^{j \frac{2 \pi}{T} n T} e^{j \varphi_{m^{\prime}, n}}$

where $a_{m^{\prime}, n}$ is a real symbol mapped from complex $\mathrm{C}_{\mathrm{m}, \mathrm{n}}$ with $\mathrm{m}$ varying from 0 to $\mathrm{M}-1$, as follows

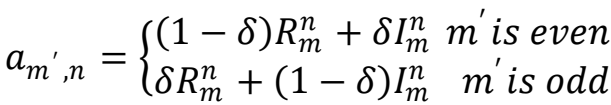

Where $\delta \epsilon\{0,1\}$ is defined as n modulo 2 .

Also, $h(t)$ is the impulse response of the prototype filter. The mathematical significance of this time-staggering rule appears in that has got a phase term $\varphi_{m^{\prime}, n}$ which is set to be $\frac{\pi}{2}\left(m^{\prime}+\right.$ $n)-\pi m^{\prime} n$ without loss of generality. PHYDYAS filter was originally designed by Bellanger and later it was chosen to be the reference prototype filter of the European project PHYDYAS. In this paper, we have chosen to use PHYDYAS filter as the prototype filter. The design of this filter is based on frequency sampling technique. The analytical parameters involved in this filter design are the number of Sub-carriers N, the overlapping factor $\mathrm{K}$, the roll-off and the length of the filter, $\mathrm{L}=\mathrm{KN}$ with desired values $\mathrm{F}(\mathrm{k}=\mathrm{L})$. where $\mathrm{k}=0,1, \mathrm{~L}-1$.

Fo $=1, F_{1}=0: 97196 ; \quad F_{2}=1 / \sqrt{2}$ (5)

$\mathrm{F}_{3}=\sqrt{1-F_{1}^{2}} \mathrm{~F}_{\mathrm{k}}=0 \quad 4<\mathrm{k}<\mathrm{L}-1$

The impulse response of the PHYDYAS filter is given as

$$
\mathrm{h}(\mathrm{t})=\left\{\begin{array}{cc}
\frac{1}{\sqrt{A}}\left[1+2 \sum_{1}^{K-1}\left(-1^{k}\right) F_{K} \cos \left(\frac{2 \pi k t}{K T}\right)\right] t \epsilon[0, K T] \\
0 & \text { elsewhere }
\end{array}\right.
$$

where the normalization constant $\mathrm{A}=\mathrm{KT}\left[1+2 \sum_{k=1}^{K-1} F_{k}^{2}\right.$

In Fig. 2, we can have a glance of the impulse responses for both OFDM which has a rectangular shape and FBMCOQAM that uses a PHYDYAS filter. We can notice that unlike the OFDM, the impulse response of the PHYDYAS filter has duration that spans over $4 \mathrm{~T}$.

\subsection{PAPR Analysis}

The PAPR is a random variable that is an appurtenant parameter in measuring the sensitivity of a non-linear HPA when a non-constant envelop input need to be transmitted. The PAPR of the continuous-time base-band signal $s(t)$, transmitted during a symbol period $\mathrm{T}$ is defined by-

$$
\operatorname{PAPR}_{S(T)}=\frac{\max _{0 \leq t \leq T}\left|s(t)^{2}\right|}{\frac{1 \int_{0}^{T}\left|s(t)^{2}\right|}{T} d(t)}
$$

The Complementary Cumulative Density Function (CCDF) of PAPR is a useful parameter to analyze the PAPR, which is defined as the probability that the PAPR of the discrete-time signal exceeds beyond a given threshold that is denoted by $\gamma$ and thereby it can be evaluated as $P_{r}\left\{P A P R_{S(n)} \geq \gamma\right\}$.

\subsection{PAPR Analysis based on Dispersive SLM}

In classical SLM scheme for OFDM, every symbol is rotated with one of a kind phase rotation vectors which are i.i.D and the optimally rotated symbol is chosen among others, based on the least PAPR criterion. The input symbol is phase rotated by $\mathrm{U}$ vectors of measurement $\mathrm{LN}$ each. These $\mathrm{U}$ rotated input symbols lift same information and pose identical constellation like the normal input symbols. After the modulation, the PAPR is calculated over an OFDM symbol interval and the symbol with the least PAPR is chosen, which is referred as optimally rotated symbol. Much like the classical SLM scheme, every symbol is rotated with distinctive section rotation vectors which can be i.I.D and the optimally rotated symbol is chosen among others headquartered on least PAPR criterion that has been computed over $[0 ; 4 \mathrm{~T})$ rather of $[0 ; \mathrm{T})$ The proposed scheme deals with the time dispersive nature of the FBMC-OQAM signals and therefore termed as Dispersive SLM. This scheme can keep for any prototype filter equivalent to IOTA (Isotropic Orthogonal Transform Algorithm)

The algorithm of DSLM scheme involves the following steps-

Step 1 - Initialization: Firstly, we generate U phase rotation vectors of length $\mathrm{N}$ as:

$$
\begin{aligned}
& \theta_{k}^{(u)}=e^{j \varphi k} \in C^{N}, \mu \in \\
& \{1, \ldots \ldots . U\}, k \in\{1 \ldots \ldots \ldots N\}, \varphi_{k} \in[0,2 \pi)
\end{aligned}
$$

Step 2- Rotation: Each input symbol vector $\mathrm{Cm}$ is then phase rotated with $\mathrm{U}$ different input vectors giving $c_{m}^{(u)}$ :

$$
C_{m}^{(u)}=C_{m} \cdot \theta^{u}
$$

where $=$ implies carrier-wise point-to-point multiplication. Likewise the symbols, $a_{m^{\prime}, n}^{u}$ are chosen from $C_{m}^{(u)}$.

Step3- Modulation: FBMC-OQAM modulation of the mth input symbol considering the overlap of previous symbols:

$$
\begin{aligned}
& S^{(u)}(t)=\sum_{n=0}^{N-1} \sum_{m=0}^{2 m-1} a_{m^{\prime}, n}^{(u m i n)} h\left(t-\frac{m^{\prime} T}{2}\right) e^{j \frac{2 \pi}{T} n t} e^{j \varphi m^{\prime}, n} \\
& +\sum_{n=0}^{N-1} \sum_{m^{\prime}=2 m}^{2 m+1} a_{m^{\prime}, n}^{(u m i n)} h\left(t-\frac{m^{\prime} T}{2}\right) e^{j \frac{2 \pi}{T} n t} e^{j \varphi_{m^{\prime}, n}}
\end{aligned}
$$

Where

(i) $S^{(u)}(t) \neq 0$ from $t=\left[0,(2 m+1) \frac{T}{2}+4 T\right)$,

(ii)

$a_{m^{\prime}, n}^{(\text {umin })}$ are from previous selected symbols $C_{m}^{(\text {umin ) }}$,

(iii) $m \in\{0,1, \ldots \ldots . M-1\}$

Step 4-PAPR Calculation: We compute the PAPR of $s^{(u)}(t)$ on a certain interval $T_{o}$ :

$\operatorname{PAPR}_{(T o)}^{(u)}=\frac{\max _{t \in T o}\left|s^{(u)}(t)\right|^{2}}{\frac{1}{T_{o}} \int\left|s^{(u)}(t)\right|^{2} d t}$

where To is any arbitrary interval that includes [mT; mT+4T] interval i.e. over $4 \mathrm{~T}$ instead of $4.5 \mathrm{~T}$ since almost all of the symbol energy lies within 4T interval.

Step 5-Selection- Among $P A P R_{T o}^{(u)}$, the index u is chosen for the signal with least PAPR as per the below criterion:

$u_{\min }=\min _{0 \leq u \leq U-1} P A P R_{(T o)}^{(u)}$ 
Step 6- Updation: We update the current overlapping input symbol vector:

$C_{m}^{\left(u_{\min }\right)}=C_{m} \cdot \theta^{\left(u_{\min }\right)}$

The input symbol that has been rotated with $\theta^{(\text {umin })}$ is considered to be optimally rotated with respect to DSLM scheme. The index $u_{\min }$ will be stored in a vector $U_{S I}$ in order to be transmitted as side information (SI) for perfect recovery of the signal at the receiver. The vector $U_{S I}$ which is a null vector initially; will be updated for every $m^{\text {th }}$ symbol by row concatenation with the newly obtained $u_{\text {min }}$, as follows

$$
U_{S I=}\left[U_{S I} u_{\min }\right]
$$

Then go to step 2 for repeating the same for the next input symbol vector $C_{m+1}$.

\section{SIMULATION RESULTS \\ 5.1 RESULT I}

The figure 3 shows the PAPR of the FBMC modulation system which is used for defining the $5 \mathrm{G}$ standards more commonly. The visualization of the figure will be well indicating the importance of DSLM technique which is rather an unconventional method of minimizing the PAPR to the lowest level so far possible, in this figure it is clear from the range of $\mathrm{x}$ axis that $\mathrm{z}$ values differ from 3 to 4.6 and similarly yaxis differs between $1 \mathrm{e}^{0}$ to $1 \mathrm{e}^{-2}$. The most important thing observed in the current discussion remains about the comparison in which original and SLM technique has shown almost same level of PAPR at around 4.4 to $4.6 \mathrm{z}$ values of PAPR but for the same SLM and DSLM has been convincing the PAPR to 3.2 to 3.6 zvalues. Thus after the complete results there is one more point that can be noticed that the current SLM and DSLM technique ranges to a very low $\mathrm{z}$ values which has'nt been observed yet in the previous paper of Reduction of PAPR for FBMC-OQAM Systems using Dispersive SLM Technique authored by S. S. Krishna Chaitanya. As far as the techniques implementations are there all of them has been calculated using MATLAB only, thus this research strongly supports and endorse use of DSLM technique authored earlier as well for FBMC OQAM systems.

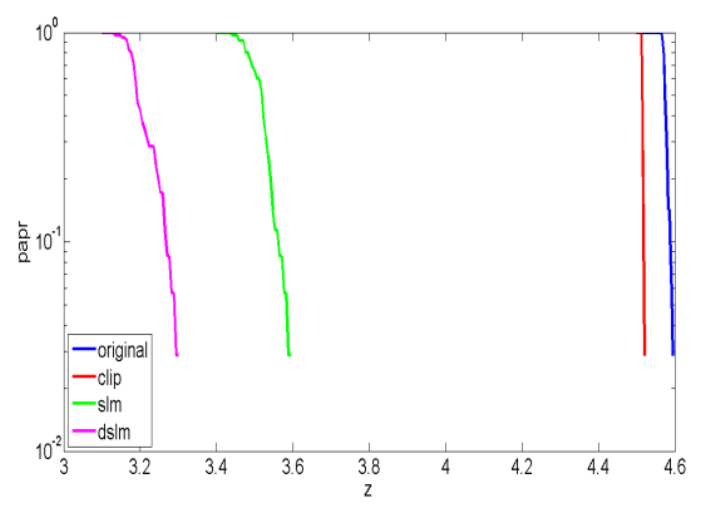

Fig 3. Peak to average power ratio of FBMC OQAM Transmitter comparing various PAPR techniques

\subsection{RESUIT II}

The following figure shows the PAPR ratio of the FBMCOQAM Transmitter using DSLM Technique. In the figure 3 the PAPR value of DSLM technique works very well as it starts nearly $1 \mathrm{~dB}$ with $\mathrm{z}$ value reaching $3.1 \mathrm{dBs}$ and the PAPR value reaching a minimum of $0 \mathrm{dBs}$ at value of $3.3 \mathrm{dBs}$ of $\mathrm{z}$.

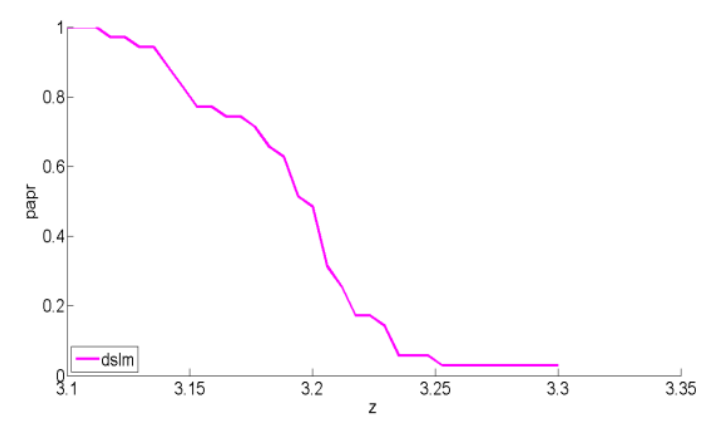

Fig 4. PAPR Ratio of FBMC -OQAM Transmitter using DSLM technique

\subsection{RESULT III}

The following figure shows the PAPR ratio of FBMC-OQAM transmitter. The two PAPR techniques used are showing the reduction in the PAPR of transmitter. This figure shows the improvement and reduction in PAPR using DSLM technique. DSLM technique is superior and better than SLM technique. The $\mathrm{x}$ axis range from 3 to 3.8 and $\mathrm{y}$ axis range from 0 to 1 . As the results of PAPR technique for both the methods are nearly reaching OdBs DSLM proves to be better contender as the value of PAPR for SLM technique reaches 0 at $\mathrm{z}$ value 3.6dB however the value of PAPR in case of DSLM technique reaches a minimum of $0 \mathrm{~dB}$ at a $\mathrm{z}$ value of $3.3 \mathrm{~dB}$, thus DSLM technique has a far more better results.

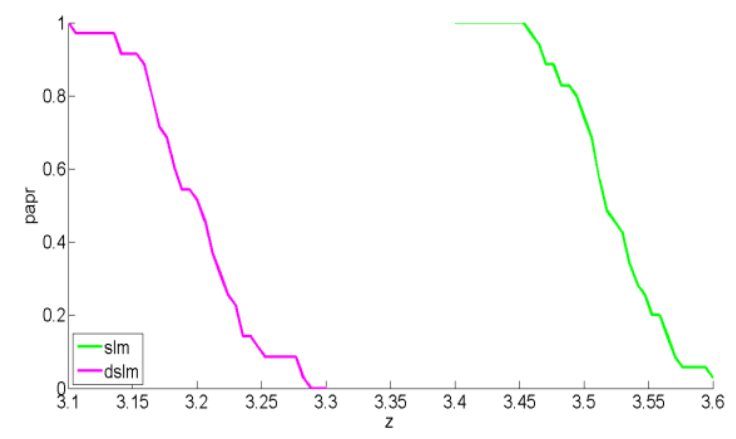

.Fig 5. PAPR ratio of FBMC-OQAM transmitter using SLM and DSLM techniques

\section{CONCLUSION}

This research work is carried out by presuming the standards of 5GNow, 4G Americas, Emphatic. All these communities and forums are appreciating the technological advancements that are endorsed by their respective governments and companies. In the current work which provides details of some different contenders of 5G technical communication systems includes FBMC, UFMC, GFDM and also their respective forums, there is also a remarkable comparison of the different forums accomplishments. In the end it is also necessary one communication methodology to be used for the achievement of some standardized results that includes Peak to average ratio calculated at the transmitter of any possible communication systems, as the results are calculated are meant for 5G FBMC systems the calculation precisely shows the equivalence and nearly same behaviour of other PAPR techniques with respect to the other systems. DSLM technique is a major technique in $5 \mathrm{G}$ systems which offers incredible reduction of power consumption at the transmitter and thus is widely used and accepted technique for FBMC technique yet it cannot be concluded that whether PAPR reduction will not hamper the data rate so this techniques alludes the fact of 
being the best technique for power reduction since bit error rate should not be affected.

\section{ACKNOWLEDGMENT}

The work done in this research work is the most substantial attainment. Special thanks to all the faculty members of Harcourt Butler Technical University, Kanpur for their guidance and support.

\section{REFERENCES}

[1]4G_Americas_5G_Spectrum_Recommendations_White_P aper-2015

[2]P. Popovski, V. Braun, H.-P. Mayer et al "ICT -317669METIS/D 1.1 V I Scenarios, requirements and KPls for $5 G$ mobile and wireless system," Tech. Rep., May 2013. [Online]. Available: https://www.metis2020.com/wpcontent/uploads/deliverables/

[3]5G wikipedia page. [Online]. Available: http://en.wikipedia.org/wiki/5G

[4].J G. Andrews, S. Buzzi, et al "What will 5G be?" IEEE J. Sel. Areas Commun. vol. 32, pp. 1065-1082, Jun. 2014.

[5] The 5G Infrastructure Public Private Partnership. [Online]. Available: http://5g-ppp.eu/

[6.] FP7 Integrating Project METIS (ICT 317669). [Online]. Available: https://www.metis2020.com/

[7.] A. Osseiran, F. Boccardi, et al, "Scenarios for 5G mobile andwireless communications: the vision of the METIS project," IEEECommun. Mag., vol. 52, pp. 26-35, May 2014.

[8]. G. Wunder, P. Jung, et al "5GNOW: non-orthogonal, asynchronous waveforms for future mobile applications," IEEE Commun. Mag., vol. 52, pp. 97-105, Feb. 2014.
[9.] FP7 STReP project 5GNOW (ICT 318555). [Online]. Available: http://www.5gnow.eu/

[10.] FP7 STReP project EMPhAtiC (ICT 318362). [Online]. Available: http://www.ict-emphatic.eu/

[11] Farhang-Boroujeny, B., "OFDM Versus Filter Bank Multicarrier," Signal Processing Magazine, IEEE vol.28, no.3, pp.92,112, May 2011.

[12]Bellanger, M., "Physical layer for future broadband radio systems,"Radio and Wireless Symposium (RWS), 2010 IEEE ,vol., no., pp.436,439, 10-14 Jan. 2010.

[13] Ndo, G.; Hao Lin; Siohan, P., "FBMC/OQAM equalization:Exploiting the imaginary interference," Personal Indoor and Mobile Radio Communications (PIMRC), 2012 IEEE 23rd International Symposium on, vol., no., pp.2359,2364, 9-12 Sept. 2012

[14] .P. Siohan and C. Siclet and N. Lacaille, "Analysis and design of OFDM/OQAM systems based on filter bank theory, IEEE Trans. onSignal Processing, vol. 50, pp. 1170-1183, May 2002.

[15]. B. L. Floch, M. Alard and C. Berrou,"Coded orthogonal frequency division multiplex, IEEE Proceedings, vol. 83, no. 6, pp. 982-996, 1995

[16]Slideshare.net [Online] Available https://www.slideshare.net/gokilabharathi/1g-vs-2g-vs-3

[17] S. S. Krishna Chaitanya BULUSUy, et al CEDRIC/LAETITIA, CNAM, Paris, France. Innov'Com, Sup'Com, Carthage University, Tunis, Tunisia."Reduction of PAPR of FBMC-OQAM systems using Dispersive SLM technique",IEEE Conference Publications,pp 568-572, 2014 